\title{
Adsorption of polymeric lattice fluids at a noninteracting hard wall : a comparison of discretized polymer-reference interaction site model (RISM) theory, Scheurtjens-Fleer theory, and Monte Carlo simulations
}

Citation for published version (APA):

Janssen, R. H. C., Nies, E. L. F., \& Cifra, P. (1997). Adsorption of polymeric lattice fluids at a noninteracting hard wall : a comparison of discretized polymer-reference interaction site model (RISM) theory, Scheurtjens-Fleer theory, and Monte Carlo simulations. Langmuir, 13(10), 2784-2790. https://doi.org/10.1021/la9608932

DOI:

$10.1021 / \mathrm{la} 9608932$

Document status and date:

Published: 01/01/1997

Document Version:

Publisher's PDF, also known as Version of Record (includes final page, issue and volume numbers)

Please check the document version of this publication:

- A submitted manuscript is the version of the article upon submission and before peer-review. There can be important differences between the submitted version and the official published version of record. People interested in the research are advised to contact the author for the final version of the publication, or visit the $\mathrm{DOI}$ to the publisher's website.

- The final author version and the galley proof are versions of the publication after peer review.

- The final published version features the final layout of the paper including the volume, issue and page numbers.

Link to publication

\footnotetext{
General rights

- You may freely distribute the URL identifying the publication in the public portal. follow below link for the End User Agreement:

www.tue.nl/taverne

\author{
Take down policy \\ If you believe that this document breaches copyright please contact us at: \\ openaccess@tue.nl \\ providing details and we will investigate your claim.
}

Copyright and moral rights for the publications made accessible in the public portal are retained by the authors and/or other copyright owners and it is a condition of accessing publications that users recognise and abide by the legal requirements associated with these rights.

- Users may download and print one copy of any publication from the public portal for the purpose of private study or research.

- You may not further distribute the material or use it for any profit-making activity or commercial gain

If the publication is distributed under the terms of Article 25fa of the Dutch Copyright Act, indicated by the "Taverne" license above, please 


\title{
Adsorption of Polymeric Lattice Fluids at a Noninteracting Hard Wall: A Comparison of Discretized Polymer-Reference Interaction Site Model (RISM) Theory, Scheutjens-Fleer Theory, and Monte Carlo Simulations
}

\author{
R. H. C. J anssen and E. Nies* \\ Department of Polymer Technology, Eindhoven University of Technology, P.O. Box 513, \\ $5600 \mathrm{MB}$ Eindhoven, The Netherlands \\ P. Cifra
}

Polymer Institute, Slovak Academy of Sciences, 84236 Bratislava, Slovakia

Received September 16, 1996. In Final Form: March 4, $1997^{\circledR}$

\begin{abstract}
A discretized version of the polymer-reference interaction site model (RISM) theory is investigated on its capabilities to assess the adsorption behavior of interacting polymeric lattice fluids near a flat wall. Both the density profile of the fluid at the wall and the equation of state of the bulk fluid (obtained from the density profile via a method of Dickman) are compared to M onte Carlo simulations. The profiles are also compar ed totheoutcomeof a classical lattice-based adsorption theory, theScheutjens-F leer formalism. At all bulk packing fractions, the Scheutjens-Fleer theory is found to be superior to the polymer-RISM theory, although it is made plausible that the performance of the discretized polymer-RISM theory can be improved significantly by using a nonreversal random walk intramol ecular distribution, instead of the random walk intramolecular distribution used in this work.
\end{abstract}

\section{Introduction}

Polymer adsorption is an interesting phenomenon with wide applications in, e.g., the composite and coatings industry. An understanding of the molecular origins of theadsorption phenomenon is of importancein optimizing processes in these industries.

Classical approaches to study polymer adsorption build on work performed on bulk lattice ${ }^{1,2}$ and continuum fluids. ${ }^{3,4}$ Well-known lattice-based adsorption theories are those of Helfand ${ }^{5,6}$ and Scheutjens and Fleer. ${ }^{7-9}$ An important aspect of thesetheories is that they account for the changes in conformational entropy of the molecules in the interfacial region. Well-known theories that consider the adsorption of a continuum space polymeric fluid at a sol $\mathrm{id}$ wall arethetheories of $\mathrm{H}$ elfand ${ }^{10}$ and $\mathrm{H}$ ong and Nool andi. ${ }^{11}$ These continuum approaches use Gaussian random walk (GRW) chain statistics to describe the polymer chain conformations in the interfacial region. GRW chain statistics apply in the bulk of dense onecomponent fluids ${ }^{12}$ but are not suited for situations that deviatestrongly from the bulk circumstances. Hence, the theories of refs 10 and 11 are less suited in cases where strong wall-fluid interactions causetheinterfacial region to be very different from the bulk of the fluid.

* Author to whom correspondence should be addressed.

$\otimes$ Abstract published in AdvanceACS Abstracts, April 15, 1997.

(1) Flory, P. J . Principles of Polymer Chemistry; Cornell University Press: Ithaca, NY, 1953.

(2) Huggins, M. L. J . Chem. Phys. 1941, 9, 440.

(3) Edwards, S. F. Proc. Phys. Soc. London 1965, 85, 613.

(4) Freed, K. F. Renormalization Group Theory of Macromolecules; Wiley-Interscience: New York, 1987.

(5) Helfand, E. J . Chem. Phys. 1975, 63, 2192.

(6) Helfand, E. Macromolecules 1976, 9, 307.

(7) Scheutjens, J. M. H. M.; Fleer, G. J . J . Phys. Chem. 1979, 83, 1619.

(8) Theodorou, D. N. Macromolecules 1988, 21, 1400.

(9) Evers, O. A. Statistical Thermodynamics of Block Copolymer Adsorption. Ph.D. Thesis, Wageningen Agricultural University, Wageningen, The Netherlands, 1993.

(10) Helfand, E. J . Chem. Phys. 1975, 62, 999.

(11) Hong, K. M.; Noolandi, J. Macromol ecules 1981, 14, 1229.

(12) Flory, P. J . J . Chem. Phys. 1949, 17, 303.
In this paper weinvestigatean approach tothepolymer adsorption phenomenon that does not build on the work of refs $1-4$. It is based on integral equations that describe the correlations of the fluid particles with the adsorbing wall. Work along this line has been presented previously in refs 13-16 in which the adsorption of monatomic spherical particles was considered. Only the integral equation study of Yethiraj and $\mathrm{Hall}^{17}$ considered the adsorption of large flexible polymeric molecules. Their method tocal culatetheadsorption profiles of a continuum space polymeric fluid near a solid impenetrable wall is based on the polymer-RISM theory. 18,19 This theory is primarily designed to explain the packing effects present in densecontinuum spacefluids. Yethiraj and $\mathrm{Hall}$ indeed found that their predictions for athermal systems are in excellent agreement with Monte Carlo (MC) simulations provided the bulk density was high enough to dominate the ordering of the fluid near the wall, i.e., in the absence of a depletion layer. In the case where depletion is important, i.e., for high molecular weight fluids or at lower bulk density, better results for theadsorption profiles are obtained via the off-lattice self-consistent field (SCF) theory of Björling and Linse. ${ }^{20}$ However, this SCF theory only captures qualitatively the layering ("packing") that occurs at high densities.

In the work presented here we apply the method of Yethiraj and $\mathrm{H}$ all to calculate the adsorption profiles, i.e., the fluid density as a function of distance from the wall, of a cubic lattice fluid. This al lows for direct comparison tothe results obtained with a classical SCF lattice model, the Scheutjens-Fleer adsorption theory. ${ }^{7}$ Furthermore, we have compared the profiles to Monte Carlosimulation data at constant number of particles, pressure, and

(13) Henderson, D.; Abraham, F. F.; Barker, J . A. Mol. Phys. 1976, 31, 1291.

(14) Henderson, D.; Plischke, M. J. Phys. Chem. 1988, 92, 7177.

(15) Zhou, Y.; Stell, G. J . Chem. Phys. 1988, 89, 7010.

(16) Schlijper, A. G.; Smit, B. Fluid Phase Equilib. 1992, 76, 11.

(17) Yethiraj, A.; Hall, C. K. J . Chem. Phys. 1991, 95, 3749.

(18) Schweizer, K. S.; Curro, J. G. Phys. Rev. Lett. 1987, 58, 246.

(19) Chandler, D.; Andersen, H. C. J . Chem. Phys. 1972, 57, 1930.

(20) Bjö rling, M.; Linse, P. J . Chem. Phys. 1992, 97, 6890. 
temperature (NpT). We have also used the adsorption profiles obtained with the integral equation formalism to obtain an equation of state (EOS) of the bulk fluid via a method of Dickman. ${ }^{21}$ The results for the E oS are also compared to simulation data.

The outline of the rest of this paper is as follows. In section 2.1 the method of Henderson, Abraham, and Barker $(\mathrm{HAB})^{13}$ for introducing a flat wall in an integral equation formalism is applied to a discretized version of the polymer-RISM equation. The HAB method is particularly easy to employ in our case of a cubiclattice fluid. The principal results of section 2.1 are expressions for the adsorption profiles and isotherms of a one-component polymeric lattice fluid consisting of linear chains. In section 2.2 the solution procedure is briefly outlined. I t is particularly easy to employ and boils down to solving two equations in two unknowns via a Newton-Raphson scheme. In section 2.3 we very briefly recapitulate a method developed by Dickman to extract an EoS of the bulk lattice fluid from the adsorption profiles. I n section 3 the NpT -MC simulation is briefly considered. Thus far, we only performed simulations for 30-mers at a noninteracting wall. Fluids with segmental interaction energies of $\mathrm{u}_{\mathrm{attr}}=-0.0 \mathrm{k}_{\mathrm{B}} \mathrm{T}$ and $-0.2 \mathrm{k}_{\mathrm{B}} \mathrm{T}$ have been considered. Theadsorption profiles that areobtained from thesimulations were extracted from runs previously used to assess the bulk structural and EoS properties of the polymeric lattice fluids. 22,23 In section 4 the results for the adsorption profiles and E oS are presented, compared, and discussed. Conclusions are collected in section 5.

\section{Polymer-RISM Theory for Lattice Fluids near a Solid Wall}

2.1. Outline of the Method. In this section the discretized version of the polymer-reference interaction site model (RISM) equation, ${ }^{24}$ considered previously in refs 22 and 23, is modified to enablethe calculation of the adsorption profiles of polymeric lattice fluids at an impenetrable wall. The method ${ }^{13}$ was originally used to study the behavior of a simple continuum fluid near an impenetrable wall and was later applied to study adsorption within the polymer-RISM theory. ${ }^{17}$ The general principle of the method is as follows. First, the polymerRISM equation for a mixture of two mol eculetypes, simple monatomic particles and polymeric molecules, is written down. Secondly, the concentration of the simple fluid particles is reduced to zero to ensure that these particles do not influence each other. In a last step, the simple particles are extended in two directions such that they turn into a wall. Then, construction of the adsorption profile of the polymer molecules at the wall amounts to calculating the correlations between the wall and an average polymer segment. From the adsorption profile the amount of adsorbed material can be quantified by construction of an adsorption isotherm. Consider a mixture of two particletypes, simple monatomi cparticles that occupy exactly one lattice site and linear polymer chains. Thechains consist of s covalently bonded segments that occupy consecutive sites on a cubic lattice. The monatomic particles and the segments of the polymer molecules interact via nearest neighbor potentials given by

(21) Dickman, R. J . Chem. Phys. 1987, 87, 2246

(22) J anssen, R. H. C.; Nies, E.; Cifra, P. Submitted for publication in Macromol ecules.

(23) J anssen, R. H. C. Structureand Thermodynamics of latticefluids in Bulk and at Interfaces. Ph.D. Thesis, Eindhoven University of Technology, Eindhoven, The Netherlands, 1996.

(24) Curro, J . G.; Schweizer, K. S. Macromolecules 1987, 20, 1928.

$$
\begin{gathered}
u_{i j}(0,0,0) \rightarrow \infty \\
u_{i j}(l, m, n)=u_{a t t r, i j} \quad \text { if } I^{2}+m^{2}+n^{2}=1 \\
u_{i j}(l, m, n)=0 \text { otherwise }
\end{gathered}
$$

where $\{i, j\} \in\{1,2\}$ denote the particle type: $i=1$ corresponds toa monatomic particleand $\mathrm{i}=2$ toa segment of a polymer chain. The structural correlations present in such a mixture can be studied with the polymer-RISM equation for mixtures. It can conveniently bewritten down in matrix form, and on the 3D cubic lattice it is given by $^{18,22}$

$$
\hat{\mathbf{H}}(\mathrm{u}, \mathrm{v}, \mathrm{w})=\hat{\Omega}(\mathrm{u}, \mathrm{v}, \mathrm{w}) \hat{\mathbf{C}}(\mathrm{u}, \mathrm{v}, \mathrm{w})(\hat{\Omega}(\mathrm{u}, \mathrm{v}, \mathrm{w})+\hat{\mathbf{H}}(\mathrm{u}, \mathrm{v}, \mathrm{w}))
$$

in which $\hat{\mathbf{H}}, \hat{\Omega}$, and $\hat{\mathbf{C}}$, are $2 \times 2$ matrices with elements $\eta_{\mathrm{mi}} \eta_{\mathrm{mj}} \hat{h}_{\mathrm{ij}}(\mathrm{u}, \mathrm{v}, \mathrm{w}), \eta_{\mathrm{mi}} \delta_{\mathrm{ij}} \hat{\omega}_{\mathrm{i}}(\mathrm{u}, \mathrm{v}, \mathrm{w})$, and $\hat{\mathrm{c}}_{\mathrm{ij}}(\mathrm{u}, \mathrm{v}, \mathrm{w})$. Thesymbols $\eta_{\mathrm{m} 1}$ and $\eta_{\mathrm{m} 2}$ denote respectively the packing fractions of monatomic particles and of the segments of the polymeric molecules. The Kronecker delta, $\delta_{\mathrm{ij}}$, has a value 1 if $\mathrm{i}=$ $\mathrm{j}$ and 0 otherwise. The $\hat{h}_{\mathrm{ij}}(\mathrm{u}, \mathrm{v}, \mathrm{w}), \hat{\omega}_{\mathrm{i}}(\mathrm{u}, \mathrm{v}, \mathrm{w})$, and $\hat{c}_{\mathrm{ij}}(\mathrm{u}, \mathrm{v}, \mathrm{w})$ represent respectively the total intermolecular twoparticle correlation function, the intramolecular twoparticledistribution, and the direct correlation function. ${ }^{18}$ The $\hat{h}_{i j}(u, v, w), \hat{c}_{i j}(u, v, w)$, and $\hat{\omega}_{i}(u, v, w)$ are written in 3D Fourier space, defined by

$$
\hat{f}(u, v, w)=\sum_{i, m, n} f(l, m, n) \cos l u \cos m v \cos n w
$$

with $f=h, \omega$, or $c$. equation 3 is only valid if thesymmetry conditions $f(l, m, n)=f( \pm l, \pm m, \pm n)$ apply. The real space analogs of $\hat{h}_{i j}(u, v, w), \hat{\omega}_{i}(u, v, w)$, and $\hat{c}_{i j}(u, v, w)$ can be found via inverse transformation

$$
\begin{aligned}
f(l, m, n) & =\left(\frac{1}{2 \pi}\right)^{3} \int_{-\pi}^{\pi} \int_{-\pi}^{\pi} \int_{-\pi}^{\pi} \hat{f}(u, v, w) \times \\
& \cos l u \cos m v \cos n w d u d v d w
\end{aligned}
$$

The meaning of the real space direct correlation function, $c_{i j}(l, m, n)$, is merely mathematical, in contrast with $h_{i j}(l, m, n)$ and $\omega_{\mathrm{i}}(\mathrm{l}, \mathrm{m}, \mathrm{n})$. The total intermolecular correlation function, $h_{i j}(l, m, n)$, is related to the probability $P_{i j}(I, m, n)$ of findinga particlej at distance $(l, m, n)$ from another particle i that is part of a different molecule, via $\mathrm{P}_{\mathrm{ij}}(\mathrm{l}, \mathrm{m}, \mathrm{n})=\eta_{\mathrm{mj}^{-}}$ $\left(h_{i j}(l, m, n)+1\right)$. The $\omega_{i}(l, m, n)$ is the intramolecular twoparticledistribution function. For themonatomicpartides the only nonzero term is $\omega_{1}(0,0,0)=1$, but in the case of flexiblepolymer molecules $\omega_{2}(l, m, n)$ does givethe packing fraction of segments located at $(I, m, n)$ from an average segment bel onging to the same molecule. If the polymer molecules are considered to be random flights, which is a reasonable first approximation, ${ }^{12,24}$ then $\hat{\omega}_{2}(u, v, w)$ is given by $22,25,26$

$$
\hat{\omega}_{2, \mathrm{RF}}(\mathrm{u}, \mathrm{v}, \mathrm{w})=\frac{1-\hat{\tau}^{2}-\frac{2}{\mathrm{~s}} \hat{\tau}+\frac{2}{\mathrm{~s}} \hat{\tau}^{\mathrm{s}+1}}{(1-\hat{\tau})^{2}}
$$

in which $\hat{\tau}(\mathrm{u}, \mathrm{v}, \mathrm{w})=(\cos \mathrm{u}+\cos \mathrm{v}+\cos \mathrm{w}) / 3$ is the Fourier transform, eq 3 , of the one-jump probability

$$
\tau(\mathrm{l}, \mathrm{m}, \mathrm{n})=\frac{1}{6} \quad \text { if } \mathrm{I}^{2}+\mathrm{m}^{2}+\mathrm{n}^{2}=1
$$

(25) Chandrasekhar, S. Rev. Mod. Phys. 1943, 15, 1.

(26) Cummings, P. T.; Stell, G. J . Stat. Phys. 1983, 33, 709. 


$$
\tau(\mathrm{l}, \mathrm{m}, \mathrm{n})=0 \quad \text { otherwise }
$$

From eq 6 it is clear that only jumps between nearest neighbor sites are allowed in our model. The discretized polymer-RISM equation eq 2 can be used to study the structural properties of polymer mixtures on a cubiclattice if the monatomi c particles 1 are replaced by a second type of polymer molecules (see, e.g., refs 18 and 27-30), but in this work eq 2 is used to study the adsorption of a polymeric lattice fluid at a flat wall. For that purpose we perform the matrix multiplications in eq 2 and take the limit $\eta_{\mathrm{m} 1} \rightarrow 0$. A set of four equations results. It is given by

$$
\begin{aligned}
\hat{h}_{i j}(u, v, w)=\hat{\omega}_{i}(u, v, w) \hat{c}_{i j}(u, v, w) \hat{\omega}_{j}(u, v, w)+ \\
\eta_{m 2} \hat{\omega}_{i}(u, v, w) \hat{c}_{i 2}(u, v, w) \hat{h}_{2 j}(u, v, w)
\end{aligned}
$$

for $\{i, j\} \in\{1,2\}$. The particles of type 1 are monomers, thus from $\omega_{1}(0,0,0)=1$ and eq 3 , we have $\hat{\omega}_{1}(u, v, w)=1$. For $\mathrm{ij}=11$, eq 7 describes the correlations between the simplefluid particles in themixture. This is of nointerest to us here: the concentration of simplefluid particles $\eta_{\mathrm{m}}$ was reduced tozeroto excludeencounters of such particles, which guarantees that the other correlations in the mixture are independent of the correlations between the simple fluid particles. For $\mathrm{ij}=12$ or $21 \mathrm{eq} 7$ denotes the correlations between a simplefluid particleand a polymer segment, and finally, for $\mathrm{ij}=22$ eq 7 governs the correlations occurring between two average segments of two different polymer molecules. In that case eq 7 is identical to the discretized polymer-RISM equation. ${ }^{22-24}$ It determines the bulk structural properties of the polymericmolecules of type 2 and can besolved separately from the three other equations in eq 7 along the lines presented in refs 22 and 23 . The results for the bulk structural quantities of the polymeric fluid, $\hat{h}_{22}, \hat{c}_{22}$ and $\hat{\omega}_{2}$, are subsequently used as input quantities in the calculation of theadsorption profile of the polymericfluid from eq 7 with $\mathrm{ij}=12$. (Alternatively we can also take $\mathrm{ij}=21$.) Equation 7 (with $\mathrm{ij}=12$ ) is written in real space form with the help of eq 4 as

$$
\begin{aligned}
\mathrm{h}_{12}(l, \mathrm{~m}, \mathrm{n})=\sum_{\substack{l^{\prime}, \mathrm{m}^{\prime}, \mathrm{n}^{\prime} \\
\eta_{\mathrm{m} 2}}}\left(\omega_{22}\left(\mathrm{~h}_{2}, \mathrm{l}^{\prime}, \mathrm{m}^{\prime}, \mathrm{n}^{\prime}, \mathrm{n}^{\prime}\right)\right) \mathrm{c}_{12}\left(l-\mathrm{l}^{\prime}, \mathrm{m}-\mathrm{m}^{\prime}, \mathrm{n}-\mathrm{n}^{\prime}\right)
\end{aligned}
$$

Themonatomic particle 1 can now effectively beextended in $m$ and $n$ direction by removing the $m$ and $n$ coordinates in the arguments of $h_{12}(I, m, n)$ and $c_{12}(I, m, n)$ : only the I distanceto particle 1 remains to bea positional parameter, and therefore, the particle turns into a wall. ${ }^{13}$ Hence, from eq 8 we obtain

$$
\mathrm{h}_{12}(\mathrm{l})=\sum_{l^{\prime}, \mathrm{m}^{\prime}, \mathrm{n}^{\prime}}\left(\omega_{2}\left(\mathrm{l}^{\prime}, \mathrm{m}^{\prime}, \mathrm{n}^{\prime}\right)+\eta_{\mathrm{m} 2} \mathrm{~h}_{22}\left(\mathrm{l}^{\prime}, \mathrm{m}^{\prime}, \mathrm{n}^{\prime}\right)\right) \mathrm{c}_{12}\left(\mathrm{l}-\mathrm{l}^{\prime}\right)
$$

Note that the creation of a wall is particularly easy on the cubiclattice, which is duetotheindependence of $\mathrm{I}, \mathrm{m}$, and $\mathrm{n}$. eq 9 is the discretized version of the equation that was used in ref 17 to study the adsorption behavior of continuum space polymeric fluids. To obtain the distribution of an average polymer segment with respect tothe wall, eq 9 has to be combined with a closure equation. Here we apply the Percus-Yevick closure. In 1D form it

(27) Schweizer, K. S.; Curro, J . G. J . Chem. Phys. 1989, 91, 5069. (28) Curro, J. G.; Schweizer, K. S. Macromol ecules 1991, 24, 6736.

(29) Schweizer, K. S.; Yethiraj, A. J . Chem. Phys. 1993, 98, 9053.

(30) Honeycutt, J. D. Macromol ecules 1994, 27, 5377. is given by ${ }^{31}$

$$
\begin{gathered}
g_{12}(0)=0 \\
c_{12}(I)=g_{12}(I)\left(1-e^{\beta u_{12}(l)}\right) \quad I \geq 1
\end{gathered}
$$

where $u_{12}(I)$ is the wall-fluid interaction potential. It is given by eq 1 for $i=1$ and $j=2$ upon removal of the $m$ and $\mathrm{n}$ dependence in that equation. Note that the wallfluid interaction is of nearest neighbor type. In eq 10 , $g_{12}(I)$ denotes the segmental distribution with respect to the wall. I ts calculation is the objective of the work presented here. The $g_{12}(I)$ and $h_{12}(l)$ are related as

$$
h_{12}(I)=g_{12}(l)-1
$$

From the adsorption profile of the polymeric lattice fluid, $\eta_{\mathrm{m} 2} \mathrm{~g}_{12}(\mathrm{I})$, an adsorption isotherm, $\Gamma\left(\eta_{\mathrm{m} 2}\right)$, is constructed as

$$
\Gamma\left(\eta_{\mathrm{m} 2}\right)=\sum_{\mathrm{l}=1}^{\text {loulk }} \eta_{\mathrm{m} 2} \mathrm{~h}_{12}(l)
$$

where $I_{\text {bulk }}$ is taken sufficiently large for the summation to reach into the bulk region of the polymeric fluid. Note that $\eta_{\mathrm{m} 2} \mathrm{~h}_{12}(\mathrm{l})$ is the excess amount of adsorbed material relative to the bulk.

The method presented in this section is al so suitable to study competitive adsorption for two or more molecule types. ${ }^{32}$ In that case the number of moleculetypes in the matrix of eq 2 has to beextended, but thegeneral procedure is not changed. Herewe are only interested in a first test of the lattice formalism and we have only studied onecomponent fluids consisting of linear polymer chains.

2.2. Solution procedure. Equations 9-11 form a complete set of equations from which the adsorption profile, $\eta_{\mathrm{m} 2} \mathrm{~g}_{12}(\mathrm{l})$, and adsorption isotherm, $\Gamma\left(\eta_{\mathrm{m} 2}\right)$, can be constructed if the bulk structural properties of the polymericfluid, $\hat{h}_{22}(u, v, w)$ and $\hat{\omega}_{2}(u, v, w)$, areknown. These quantities have al ready been obtained in refs 22 and 23.

The set of eqs 9-11 are conveniently solved in 1DFourier space, defined by

$$
\tilde{f}(u)=\sum_{1} f(l) \cos l u
$$

with inverse

$$
f(I)=\frac{1}{2 \pi} \int_{-\pi}^{\pi} \tilde{f}(u) \cos l u d u
$$

With help of eq 13 we can write eq 9 as

$$
\tilde{h}_{12}(u)=\left(\hat{\omega}_{2}(u, 0,0)+\eta_{m 2} \hat{h}_{22}(u, 0,0)\right) \tilde{c}_{12}(u)
$$

where $\hat{h}_{22}(u, 0,0)$ and $\hat{\omega}_{2}(u, 0,0)$ are 3D-Fourier series, given by $\mathrm{Eq} 3$ for $\mathrm{v}=\mathrm{w}=0$. We do not have to take Fourier sine terms into account in eq 13 , because blowing up particle 1 does not violate the symmetry rel ation $h_{12}(l)=$ $\mathrm{h}_{12}(-\mathrm{I})$. I $\mathrm{n}$ eq 15 we replace $\hat{\omega}_{2}(\mathrm{u}, 0,0)+\eta_{\mathrm{m} 2} \hat{\mathrm{h}}_{22}(\mathrm{u}, 0,0)$ by $\hat{\omega}_{2}(\mathrm{u}, 0,0) /\left(1-\eta_{\mathrm{m}_{2}} \hat{\omega}_{2}(\mathrm{u}, 0,0) \hat{c}_{22}(\mathrm{u}, 0,0)\right)$ (see eq 7 for $\left.\mathrm{ij}=22\right)$, which is convenient due to the short-ranged character of the direct correlation function $\hat{C}_{22}(u, v, w)$. Combination of eq 15 with the 1D-PY closure, eq 10, thus results in two equations in the unknowns $c_{12}(0)$ and $c_{12}(1)$

(31) Percus, J . K.; Yevick, G. J . Phys. Rev. 1958, 110, 1.

(32) Yethiraj, A. Phys. Rev. Lett. 1995, 74, 2018. 


$$
\begin{aligned}
& -1=\frac{1}{2 \pi} \int_{-\pi}^{\pi} \frac{\hat{\omega}_{2}(u, 0,0)}{1-\eta_{m 2} \hat{\omega}_{2}(u, 0,0) \hat{c}_{22}(u, 0,0)} \tilde{c}_{12}(u) d u \\
& c_{12}(1) /\left(1-e^{\beta u u_{12}(1)}\right)-1= \\
& \frac{1}{2 \pi} \int_{-\pi}^{\pi} \frac{\hat{\omega}_{2}(u, 0,0)}{1-\eta_{m 2} \hat{\omega}_{2}(u, 0,0) \hat{c}_{22}(u, 0,0)} \tilde{c}_{12}(u) \cos u d u
\end{aligned}
$$

in which, from eqs 10 and $13, \tilde{c}_{12}(u)=c_{12}(0)+2 c_{12}(1) \cos$ $u$. Equation 16 is solved numerically with a combined Newton-Raphson 1D quadratureroutine. Once $c_{12}(0)$ and $\mathrm{C}_{12}(1)$ arefound, thewall-segment correlations areobtained from

$$
\begin{array}{r}
\mathrm{h}_{12}(\mathrm{l})=\frac{1}{2 \pi} \int_{-\pi}^{\pi} \frac{\hat{\omega}_{2}(\mathrm{u}, 0,0)}{1-\eta_{\mathrm{m} 2} \hat{\omega}_{2}(\mathrm{u}, 0,0) \hat{\mathrm{c}}_{22}(\mathrm{u}, 0,0)} \times \\
\tilde{\mathrm{c}}_{12}(\mathrm{u}) \cos \mathrm{lu} \mathrm{du}
\end{array}
$$

(See eqs 14 and 15.)

From eq 16, the adsorption profile of an athermal monomeric fluid is found to be

$$
\begin{gathered}
\mathrm{h}_{12}(0)=-1 \\
\mathrm{~h}_{12}(1)=-\left(\frac{\left(\eta_{\mathrm{m} 2}-1\right)\left(1-\mathrm{e}^{\beta \mathrm{u}_{12}(1)}\right)}{1+\left(\eta_{\mathrm{m} 2}-1\right)\left(1-\mathrm{e}^{\beta \mathrm{u}_{12}(1)}\right)}\right) \\
\mathrm{h}_{12}(\mathrm{l})=0 \quad \text { if } \mathrm{I} \geq 2
\end{gathered}
$$

It can be shown that this result is exact. ${ }^{23}$ Note from eq 18 that the influence of the wall reaches only one layer deep into the athermal monomeric fluid.

2.3. The Wall Equation of State. Dickman ${ }^{21,33}$ has presented a method to calculate the E oS of a polymeric latticefluid from its adsorption profileat a repulsivewall. It is an alternative to the pressure route ${ }^{34,35}$ which is not valid for lattice fluids. ${ }^{21}$ The lattice wall E oS reads

$$
\frac{\mathrm{pv}_{0}}{\mathrm{k}_{\mathrm{B}} \mathrm{T}}=\int_{\phi=0}^{1} \frac{\eta_{\mathrm{m} 2} \mathrm{~g}_{12}(\mathrm{I})}{\phi} \mathrm{d} \phi
$$

where $v_{0}$ is the volume of one lattice site and $\phi=\mathrm{e}^{-\beta \mathrm{u}_{12}(1)}$. In the derivation of eq 19, the overall potential energy of the system is divided in a bulk part containing the restrictions which define the chain connectivity and prohibit segmental overlaps, and in a part $U_{12}$ caused by thewall-fluid interactions. If $\mathrm{U}_{12}$ is further decomposed (which is possible without approximation) into separate nearest neighbor wall-segment interactions $u_{12}(1)$, one obtains eq 19. ${ }^{21}$ The integration limits $(\phi=0$ and $\phi=1)$ in eq 19 correspond respectively toa wall with an infinitely strong repulsive nearest neighbor interaction and to a noninteracting wall. Equation 19 was originally used to extract the $\mathrm{E}$ oS of a latticefluid from NVT MC simulation data. Here we use it to obtain an alternative route to the E oS within the framework of section 2.1. Results for the wall EoS will be presented in section 4 .

\section{Monte Carlo Simulations}

In previous work ${ }^{22,23}$ we reported N PT MC simulations from which thebulk two-particledistributions, $h_{22}(I, m, n)$ and $\omega_{2}(I, m, n)$, and the E oS of 30 meric cubic lattice fluids

(33) Hertanto, A.; Dickman, R. J . Chem. Phys. 1988, 89, 7577.

(34) McQuarrie, D. A. Statistical Mechanics; Harper and Row Publishers: New York, 1976.

(35) Honnell, K. G.; Hall, C. K.; Dickman, R. J . Chem. Phys. 1987, $87,664$. with segmental interaction energies of $u_{a t t r}=-0.0 k_{B} T$ and $-0.2 \mathrm{k}_{B} T$ were obtained. The simulation method is based on work by Madden ${ }^{36}$ and consi ders a polymer slab condensed between a flat wall and a solid piston. A part from the bulk properties, the method al so produces the adsorption profiles of the fluids at theflat wall. Here, we usetheseprofiles for comparison to theadsorption profiles calculated with the polymer-RISM and Scheutjens-Fleer theory. Thus far, only nonattracting walls are considered.

\section{Results and Discussion}

4.1. Adsorption Profiles. We start by making a few general remarks about the Scheutjens-Fleer (SF) theory. ${ }^{7,37,38}$ It is an extension of theFlory-Huggins mean field lattice theory ${ }^{1}$ in which a preaveraging of polymer concentrations in the layers parallel totheadsorbing wall takes place (mean field approximation). All possible molecular conformations are accounted for by a matrix method that calculates the probability of each molecular conformation to occur near the wall. The matrix method accounts for compositional differ ences between thelayers that occur due to the presence of the wall and that propagate via the chain-connectivity and segmental interaction energies. ${ }^{7}$ The SF theory, contrary to the method of section 2.1, allows for conformational changes of the adsorbing molecules. The method is called selfconsistent, for the distribution of the molecules over the layers is found by a minimization of the overall free energy of theadsorbing fluid. I ts mean field (MF) approximation is physically plausible at higher bulk packing fractions but seems invalid at lower packing fractions. Therefore, it is interesting to compare the SF theory to the approach of section 2.1, which does not adopt a MF approximation. Another reason for us to compare the abilities of the approach of section 2.1 totheSF formalism isthesimplicity of the integral equation approach: it only takes solving eq 16 to obtain $\mathrm{g}_{12}(\mathrm{I})$ via eq 17 , whereas in theSF formalism a coupling of several equations for each layer makes the numerical solution of these equations more complicated.

In this paper, we refer to the original SF formalism, ${ }^{7}$ although moresophisticated versions that usea different discretization of space or partially account for the intramolecular excluded chain volume via a rotational isomeric state (RIS) scheme ${ }^{39,40}$ exist. Note that comparison totheSF formal ism that uses a RIS schemewould bemoreappropriateif the (RF) intramol ecular distribution of eq 5 is repl aced by a nonreversal random walk (NRRW) intramolecular distribution.

In Figures 1 and 2, some adsorption profiles of linear 30 mer lattice polymeric chains at a noninteracting wall are shown. In Figure $1, \mathrm{u}_{\mathrm{attr}}=-0.0 \mathrm{k}_{\mathrm{B}} \mathrm{T}$ and $\eta_{\mathrm{m} 2}$ is 0.2074 $(\bullet$, and lower lines) and 0.6865 ( $\bullet$, and upper lines). In Figure $2, \mathrm{u}_{\mathrm{attr}}=-0.2 \mathrm{k}_{\mathrm{B}} \mathrm{T}$ and $\eta_{\mathrm{m} 2}$ is 0.2940 (•, lower lines) and $0.7262(\bullet$, and upper lines). Thesymbols in thefigures are obtained from MC simulations, the dashed lines from theSF theory, and the full lines from the method outlined in section 2.1. Wehavedrawn linesthrough thecalculated profiles todistinguish between theor etical and simulation data.

The MC points in Figures 1 and 2 show a depletion of polymeric molecules near the noninteracting wall in all

(36) Madden, W. G. J . Chem. Phys. 1987, 87, 1405.

(37) Fleer, G. J .; Cohen Stuart, M. A.; Scheutjens, J. M. H. M.; Cosgrove, T.; Vincent, B. Polymers at Interfaces; Chapman and Hall: London, 1993.

(38) Theodorou, D. N. Macromolecules 1988, 21, 1391.

(39) Leermakers, F. A. M.; Scheutjens, J . M. H. M. J . Chem. Phys 1988, 89, 3264

(40) Flory, P. J . Statistical Mechanics of Chain Molecules; Hanser Publishers: Munich, 1989. 


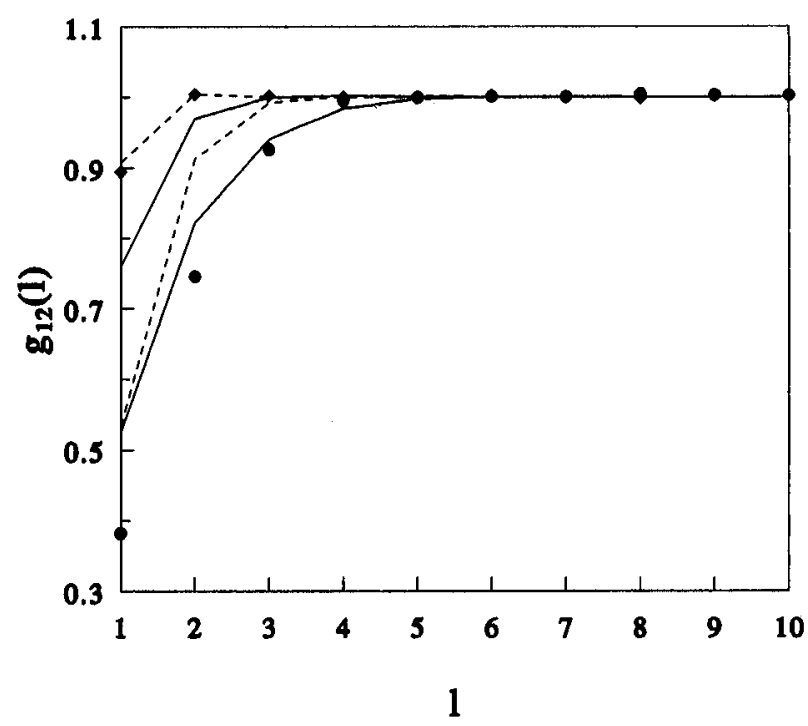

Figure 1. Adsorption profiles of an athermal 30 mer linear chain fluid at a noninteracting wall located at I $=0$. the bulk packing fractions are $\eta_{\mathrm{m} 2}=0.2074$ (๑, lower dashed and full line) and 0.6865 ( $\diamond$, upper dashed and full line). The symbols are obtained from the simulations, the dashed lines from the Scheutjens-Fleer theory, and the full lines from the polymerRISM theory of section 2.1.

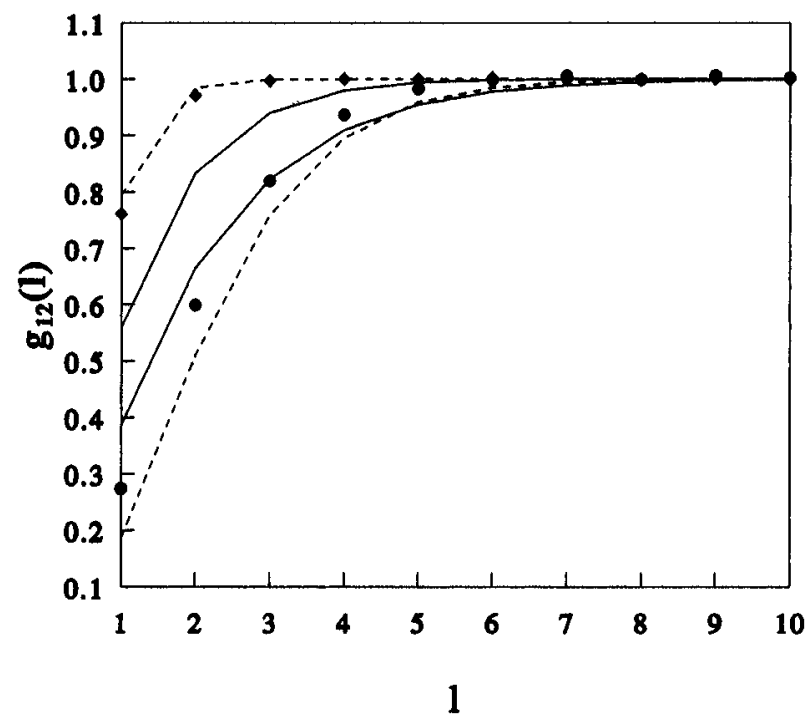

Figure 2. Caption as in Figure 1, but for $\mathrm{u}_{\text {attr }}=-0.2 \mathrm{k}_{\mathrm{B}} \mathrm{T}$ with $\eta_{\mathrm{m} 2}=0.2940$ (๑, lower dashed and full curve) and $\eta_{\mathrm{m} 2}=0.7262$ $(\diamond$, upper dashed and full line).

four cases. In Figure 1, where results for an athermal fluid near a noninteracting wall aredepicted, the depletion is of purely entropic origin: an average segment of a polymericmoleculehas a reduced probability of being close to the wall, due to chain-connectivity constraints. In Figure 2, the depletion is increased, when compared to the corresponding packing fractions in Figure 1, due to the cohesive energy of the bulk fluid: apart from the entropic restrictions that the mol ecules experience in the vicinity of thewall, thereis an additional depletion because the wall prevents the molecular segments close to the noninteracting wall to form energetically favorable $\left(u_{\text {attr }}\right.$ $=-0.2 \mathrm{k}_{\mathrm{B}} \mathrm{T}$ ) segmental interactions. Note from both figures that the depletion hole is deeper at lower bulk packing fractions: at higher bulk packing fractions the polymeric material is "pushed" to the wall, and in the limit $\eta_{\mathrm{m} 2} \rightarrow 1$, the simulations will show a compl etely flat adsorption profile, $g_{12}(I)=1$ for all I, corresponding to a complete absence of wall-fluid correlations.

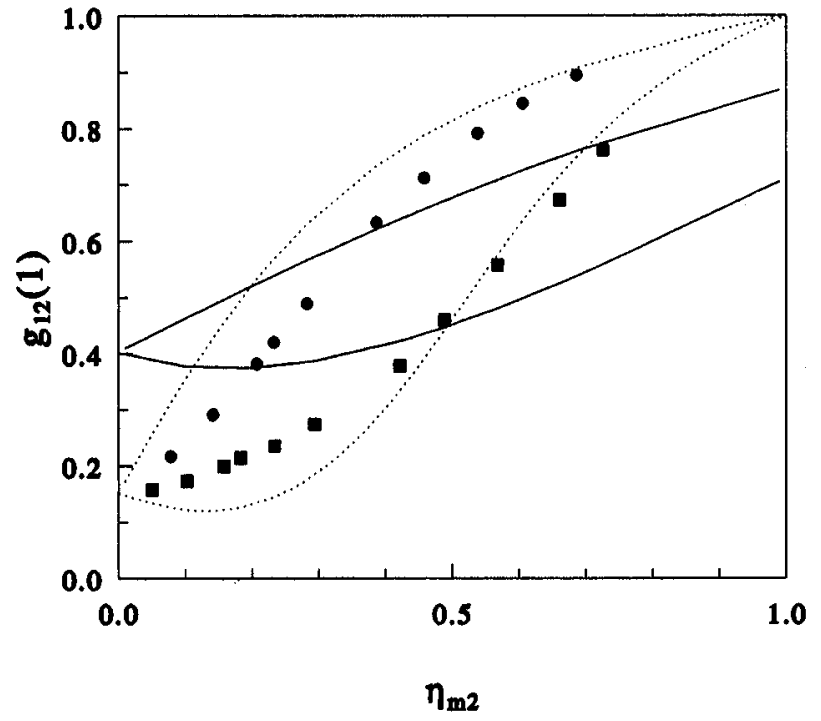

Figure 3. Bulk density dependence of the normal ized packing fraction in thelayer closest tothenonattracting wall. Full lines are obtained within the polymer-RISM theory and the dotted lines within theSF formalism of ref 7 . The symbols areobtained from MC simulation. Circles (๑) represent an athermal 30 mer fluid, and boxes (ם) a 30 mer fluid with segmental interaction strengths of $u_{\text {attr }}=-0.2 k_{B} T$. L ower full and dotted lines are for theinteracting fluid, and upper lines arefor the athermal fluid.

From the figures it is seen that the SF theory performs very good at high packing fractions (upper dashed lines), which was to be expected because the MF approximation is most appropriate for dense fluids, and still reasonably well at lower packing fractions (see also Figure 3). The polymer-RISM theory performs less well at high packing fractions: from the upper full lines in Figures 1 and 2 it is seen that for both the athermal fluid and the fluid with $\mathrm{u}_{\text {attr }}=-0.2 \mathrm{k}_{\mathrm{B}} \mathrm{T}$, the depletion at the wall is severely overestimated. Theabsence of possibilities to changethe form of themolecules in theinterfacial region will certainly be of influence on this failure, but as will becomeapparent soon, the absence of excluded volume in the RF intramolecular distribution $\hat{\omega}_{2, \mathrm{RF}}(\mathrm{u}, \mathrm{v}, \mathrm{w})$ is more important. The underestimation of the depletion at the lower packing fractions (lower full lines in Figures 1 and 2 is also due to the use of this deficient $\hat{\omega}_{2, \mathrm{RF}}(\mathrm{u}, \mathrm{v}, \mathrm{w})$, but before such is discussed we first present an overview of the behavior of the polymer-RISM and SF theory in Figure 3.

In Figure 3, we have plotted the normalized packing fraction in the layer closest to the wall, $g_{12}(l)$, versus the bulk packing fraction, of an athermal $(\bullet)$ and interacting $\left(\boldsymbol{\square}, \mathrm{u}_{\text {attr }}=-0.2 \mathrm{k}_{\mathrm{B}} \mathrm{T}\right) 30$ mer fluid for the polymer-RISM (full lines) and the SF formalism (dotted lines). The symbols are the results of Monte Carlo simulations. The upper full and dotted lines are for the athermal fluid, and the lower full and dotted line are for the interacting fluid.

First, we consider theSF results. It is seen that the $\eta_{\mathrm{m} 2}$ dependence of $g_{12}(I)$ displayed by the dotted lines is reasonably accurate for $\eta>0.5$. TheSF theory shows the physically correct full packing limit $\mathrm{g}_{12}(\mathrm{I})=1$ at $\eta_{\mathrm{m} 2}=1$. At low packing fraction $\left(\eta_{\mathrm{m} 2}<0.5\right)$ the SF theory is less accurate, although still reasonable. Weexpect that better results can be obtained by using the RIS scheme of ref 39 or by adopting a higher order MF approximation within the layers.

The predictions of the polymer-RISM theory of section 2.1 (full lines) are further off the simulation results than the SF lines in Figure 3. The full lines clearly show that the dependence of $\mathrm{g}_{12}(\mathrm{I})$ on $\eta_{\mathrm{m} 2}$ is too weak: the $\mathrm{g}_{12}(\mathrm{I})$ is overestimated at low $\eta_{\mathrm{m} 2}$ and underestimated at high $\eta_{\mathrm{m} 2}$.

The incorrect high and low density limits of $g_{12}(l)$ in the 
polymer-RISM theory are mainly due to the absence of excluded volume in the random flight intramolecular distribution function, $\hat{\omega}_{2, \mathrm{RF}}(\mathrm{u}, \mathrm{v}, \mathrm{w})$. At high packing fraction this is seen most easily. Due to the occurrence of intramolecular segmental overlaps, thereareunoccupied and doubly occupied sites, even if thelattice is fully packed with polymeric molecules. Hence, the sum of the interand intramolecular correlations in the bulk of the fluid, $\omega_{2}(\mathrm{l}, \mathrm{m}, \mathrm{n})+\eta_{\mathrm{m} 2} \mathrm{~h}_{22}(\mathrm{l}, \mathrm{m}, \mathrm{n})$, is not equal to zero, although this is the physically correct value for a fully packed lattice. ${ }^{22}$ As a result, there still is a depletion at the wall for $\eta_{\mathrm{m} 2}=1$ : it is seen from eq 9 that the physically correct adsorption profile $h_{12}(I)=0$ is only obtained if $\omega_{2}(I, m, n)$ $+\eta_{\mathrm{m} 2} \mathrm{~h}_{22}(\mathrm{I}, \mathrm{m}, \mathrm{n})=0$. In ref 22 it was shown that these "rest correlations", the deviations of $\omega_{2}(\mathrm{l}, \mathrm{m}, \mathrm{n})+\eta_{\mathrm{m} 2} \mathrm{~h}_{22^{-}}$ $(\mathrm{l}, \mathrm{m}, \mathrm{n})$ from 0 , at $\eta_{\mathrm{m} 2}=1$ can significantly be reduced by using an intramolecular distribution function, $\hat{\omega}_{2}(u, v, w)$, that (partly) excludes intramolecular segmental overlaps (e.g., the intramolecular distribution of a nonreversal random walk).

At lower packing fractions, the absence of excluded volumein $\hat{\omega}_{2, \mathrm{RF}}(\mathrm{u}, \mathrm{v}, \mathrm{w})$ is al soresponsiblefor theinaccuracy of the polymer-RISM theory. Consider a lattice fluid at bulk packing fraction $\eta_{\mathrm{m} 2} \rightarrow 0$ near a nonattracting wall. For that case, $g_{12}(\mathrm{I})$ can be expressed with help of eqs 9 and 10 as

$$
g_{12}(l)=1-\frac{\sum_{m, n} \omega_{2, R F}(1, m, n)}{\sum_{m, n} \omega_{2, R F}(0, m, n)}
$$

From eq 20 it is seen that $g_{12}(l)$ is fully determined by the bulk intramolecular distribution function. The equation also shows that the adsorption profiles of the athermal and interacting fluid display the same limiting value of $g_{12}(I)$ (full lines in Figure 3), because we have taken the same $\hat{\omega}_{2, \mathrm{RF}}(\mathrm{u}, \mathrm{v}, \mathrm{w})$ in the calculation of the adsorption profiles of both fluids. Notefrom Figure 3 that the values of $g_{12}(\mathrm{I})$ calculated from the SF theory do also meet at $\eta_{\mathrm{m} 2}$ $\rightarrow 0$. In theSF theory, this "singlechain adsorption" Iimit is determined by the wall-fluid interaction. The $g_{12}(I)$ values observed for the simulated fluids alsotend to meet at $\eta_{\mathrm{m} 2} \rightarrow 0$, although slightly different values for $g_{12}(I)$ are expected in the simulations, because the average conformations of isolated interacting and athermal chains are expected to be different. N ow, if in eq 20, we replace the random flight intramolecular distribution terms $\omega_{2, \mathrm{RF}^{-}}$ $(0, m, n)$ and $\omega_{2, R F}(1, m, n)$ by thecorresponding nonreversal random walk terms $\omega_{2, N R R W}(0, m, n)$ and $\omega_{2, N R R W}(1, m, n)$ (see refs 22 and 23 for the calculation of the $\omega_{2, \mathrm{NRRW}}$ terms), weobservea lowering of $\mathrm{g}_{12}(\mathrm{I})$ from 0.399 to 0.268 . Figure 3 shows that this is a very significant improvement of the polymer-RISM based adsorption theory.

Use of a NRRW intramolecular distribution function instead of a RF intramolecular distribution not only lowers $\mathrm{g}_{12}(\mathrm{I})$ at $\eta_{\mathrm{m} 2} \rightarrow 0$ but also increases $\mathrm{g}_{12}(\mathrm{I})$ at $\eta_{\mathrm{m} 2}=1$, as al ready discussed. Therefore, the density dependence of $g_{12}(\mathrm{l})$ is expected to becloser to the $\eta_{\mathrm{m} 2}$ dependence observed from the simulations, if a NRRW intramolecular distribution is used in thetheory of section 2.1. Thus far wehave not undertaken calculations with this improved intramolecular distribution function. In a later stage, it might al so be necessary to incorporate the effects of the wall on the average conformation of the molecules.

B eforethe equation of stateresults are examined, some words of caution concerning theapplicability of theintegral equation theory in combination with approximateclosures, such as the PY closure used here, are appropriate. For

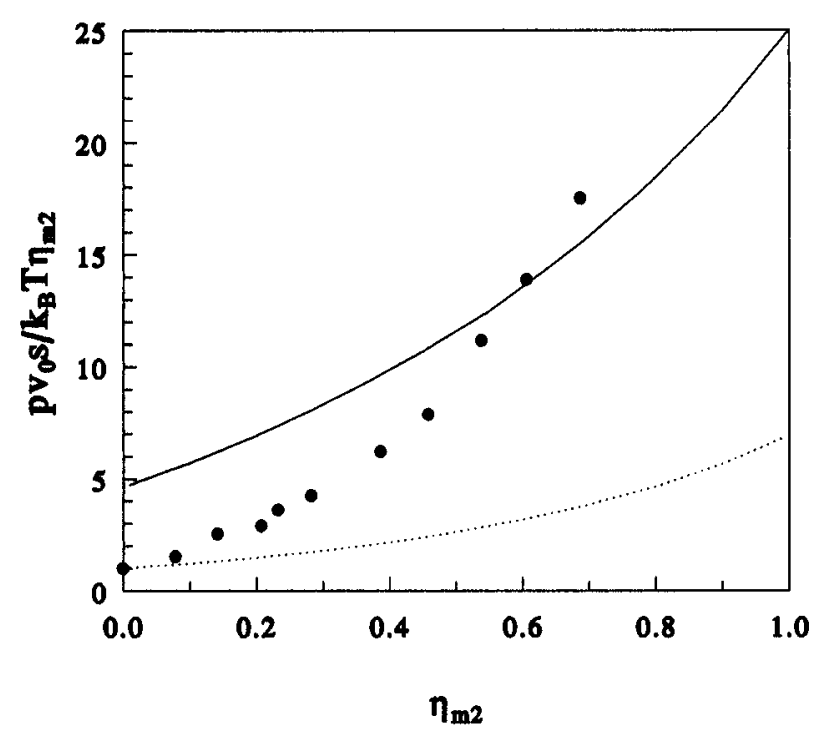

Figure 4. Compressibility factor of an athermal cubic lattice fluid consisting of RF 30 mers. Circles (0) indicate simulation results. The dotted line has been obtained previously via the compressibility-PY route (ref 22). The full line indicates the result obtained via the wall method of section 2.3.

a monatomic continuum fluid, it has been found that at a solid wall the theory is quite successful in the case of athermal fluids. However, in the case of interparticle attractive interactions, the use of the PY approximation leads to the prediction ${ }^{41}$ that the contact value at the wall obeys $\mathrm{g}_{12}(0)=(\partial \beta \mathrm{P} / \partial \eta)^{1 / 2}$, which can be considerably different from the exact result $\mathrm{g}_{12}(0)=\beta \mathrm{P} / \eta{ }^{42}$ Furthermore it has been demonstrated that integral equation theories in combination with approximate closures, such as the PY one, are unable to account for the complete wetting by liquid occurring at the solid-gas interface or thecompletewetting by gas at a purely repulsivesubstrate at the solid-liquid interface. ${ }^{43}$ Keeping this in mind one may anticipatesimilar difficulties with the polymer-RISM theory discussed here. Fortunately, in the case of polymeric fluids the occurrance of the gas-liquid transition is of no real practical consequence and these deficiencies are most likely only of minor importance.

4.2. Equation of State. In this section some results obtained with the wall EoS, eq 19, are presented and compared to MC simulations and to E oS obtained previously from the compressibility-Porcus-Yevick (c-PY) and energy-mean spherical approximation (e-MSA) route. ${ }^{22,23}$

In Figures 4and 5we have plotted the compressibility factor $\mathrm{pv}_{0} \mathrm{~s} / \mathrm{k}_{\mathrm{B}} \mathrm{T} \eta_{\mathrm{m} 2}$ obtained via MC simulation ( $(\bullet)$, the wall route (full line), the c-PY route (dotted line), and the e-MSA route (dashed line) for a 30 mer fluid with segmental interaction strengths of $u_{\text {attr }}=0$ (Figure 4), and $-0.2 \mathrm{k}_{\mathrm{B}} \mathrm{T}$ (Figure 5). The integration over $\phi$ in the wall-E oS was performed numerically and interpolates between values of $\eta(1)=\eta_{\mathrm{m} 2} \mathrm{~g}_{12}(\mathrm{I})$ calculated for 15 wallfluid interaction strengths varying from $\mathrm{u}_{12}(1) \rightarrow \infty(\phi=$ $0)$ to $u_{12}(1)=0(\phi=1)$. In Figure 4, the e-MSA E oS is not shown, because it reduces to the c-PY E oS in this case of an athermal fluid. The lines depicted in the two figures all usetherandomflight intramolecular distribution, given by eq 5. Clearly, none of the routes (c-PY, e-MSA, wall) results in an EoS that reasonably reproduces the $M C$ results, although theabsolutevalues of thecompressibility factor, $\mathrm{pv}_{0} \mathrm{~s} / \mathrm{k}_{\mathrm{B}} \mathrm{T} \eta_{\mathrm{m} 2}$ are the best for the wall EoS.

(41) Sullivan, D. E.; Stell, G. J . Chem. Phys. 1978, 69, 5450

(42) Fisher, I. Z. Statistical Theory of Liquids; University of Chicago: Chicago, IL, 1967.

(43) Evans, R.; Tarazona P.; Marini Bettolo Marconi, U. Mol. Phys. 1983, 50, 993. 


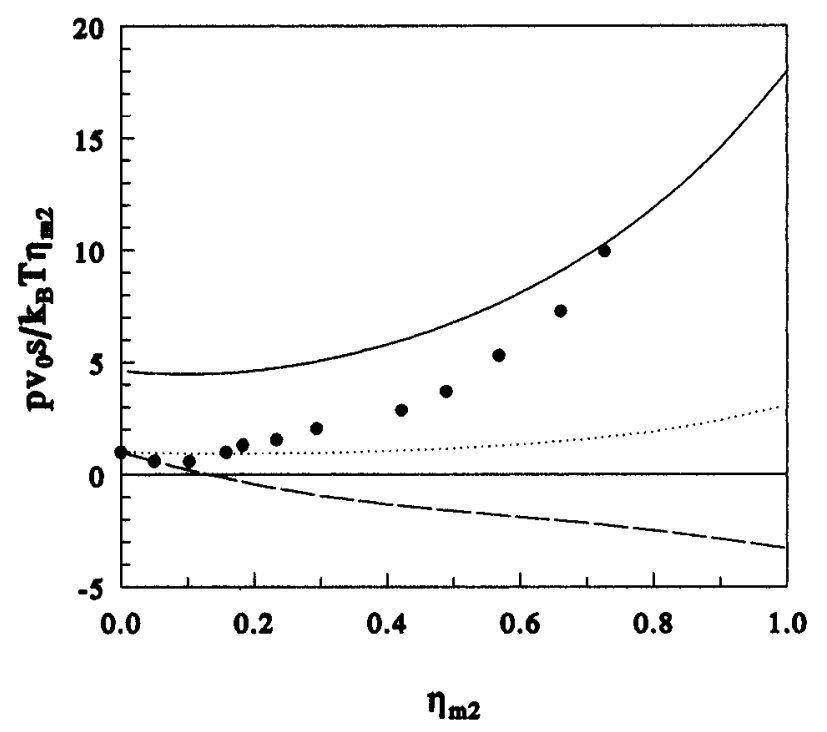

Figure 5. Compressibility factor of a cubic lattice fluid consisting of 30 mer RF chains with segmental interactions of $\mathrm{u}_{\text {attr }}=-0.2 \mathrm{k}_{\mathrm{B}} \mathrm{T}$. The circle $(\bullet)$ is obtained from simulations. Thedashed and dotted lines indicateresults obtained previously via respectively theenergy-MSA and compressi bility-PY route. The full line indicates the wall EoS obtained via eq 19.

Thec-PY and e-MSA E oS have been discussed in detail elsewhere.22,23 There it was found that these EoS are extremely sensitivetotheexact form of theintramolecular distribution function that is used. The c-PY and e-MSA E oS show the correct ideal gas limit, $\mathrm{pv} \mathrm{v}_{0} \mathrm{~S} / \mathrm{K}_{\mathrm{B}} \mathrm{T} \eta_{\mathrm{m} 2}=1$ for $\eta_{\mathrm{m} 2} \rightarrow 0$. This is in contrast to the wall EoS which, not surprisingly, does not show this limit. This is a result of the absence of excluded chain volume in $\hat{\omega}_{2, \mathrm{RF}}(\mathrm{u}, \mathrm{v}, \mathrm{w})$. It was shown in the previous subsection that if $\hat{\omega}_{2, \mathrm{RF}}(\mathrm{u}, \mathrm{v}, \mathrm{w})$ is used, then $\mathrm{g}_{12}(\mathrm{I})$ is overestimated at $\eta_{\mathrm{m} 2} \rightarrow 0$ (seeF igure 3). Such will also be the case for a repulsive wall, and hence, the compressi bi lity factor calculated from $g_{12}(I)$ via eq 19 will be overestimated at $\eta_{\mathrm{m} 2} \rightarrow 0$. Therefore, an improved intramolecular distribution function will certainly lead to an improved compressibility factor at $\eta_{\mathrm{m} 2}$ $\rightarrow 0$. The physically correct ideal gas limit will not be obtained easily, because in that $\operatorname{case}_{12}(\mathrm{I})$ has to beknown exactly. Note from eq 18 that in the case of a monomeric fluid, $g_{12}(I)$ attains the value $g_{12}(I)=e^{-\beta u_{12}(1)}$ in the limit $\eta_{\mathrm{m} 2} \rightarrow 0$. Thus, insertion of $g_{12}(I)$ in the wall EoS does, in the case of a monomeric fluid, indeed produce the correct ideal gas limit $\mathrm{pv}_{\mathrm{o}} /\left(\mathrm{k}_{\mathrm{B}} \mathrm{T} \eta_{\mathrm{m} 2}\right)=1$.

From Figures 4 and 5 it is al so seen that the pressure calculated from the wall EoS does not rise to infinity at $\eta_{\mathrm{m} 2} \rightarrow 1$, again duetotheuse of $\hat{\omega}_{2, \mathrm{RF}}(\mathrm{u}, \mathrm{v}, \mathrm{w})$ : the physically correct adsorption profile, $g_{12}(I)=1$, for which the compressibility factor does go to infinity (see eq 19), will only be obtained if there are no "rest correlations" at $\eta_{\mathrm{m} 2}$ $\rightarrow 1$, thus if $\omega_{2}(\mathrm{l}, \mathrm{m}, \mathrm{n})+\eta_{\mathrm{m} 2} \mathrm{~h}_{22}(\mathrm{I}, \mathrm{m}, \mathrm{n})=0$ for all $(\mathrm{I}, \mathrm{m}, \mathrm{n})$ (see eq 9). As shown previously, ${ }^{22}$ reductions of the "rest correl ations", and thus improvements of thehigh-density limit of the E OS, are obtained if $\hat{\omega}_{2, \mathrm{RF}}(\mathrm{U}, \mathrm{v}, \mathrm{w})$ is replaced by $\hat{\omega}_{2, \mathrm{NRRW}}(\mathrm{u}, \mathrm{v}, \mathrm{w})$. Notefrom eq 18 that monomeric fluids do again behave correctly at a fully packed lattice: $g_{12}(I)=$ 1 , and from eq 19 it is then seen that the pressure does rise to infinity for a fully packed monomeric lattice fluid.

\section{Conclusions}

In this work we have obtained the adsorption profiles of polymeric lattice fluids near an impenetrable wall via a polymer-RISM theory based approach. Calculated profiles for 30 mer fluids near a noninteracting wall have been compared to N pT Monte Carlo simulations and to the Scheutjens-Fleer (SF) theory. ${ }^{7}$ The results of the comparison of both theories to the simulations indicate that the SF theory is more accurate than the polymerRISM based theory at all packing fractions.

We have al so obtained results for the E oS of the bulk fluid from theadsorption profiles. ${ }^{21}$ TheE oS results have been compared to simulation results and to previously obtained results based on the compressibility-PY and energy-MSA route.22,23

The absolute values of the compressibility factor obtained via thewall E OS areshown to bean improvement over the values calculated via the compressibility-PY and energy-MSA route, although thewall E oS does not display the correct high and low density limits.

It is shown that thereason for theobserved deficiencies of the theory can be largely attributed to the absence of excluded volume in the intramolecular distribution function that was employed. It is made plausible that an improved intramolecular distribution function, e.g., a NRRW scheme, will result in improved adsorption profiles and E oS. Such improvements are straightforward, and since the adsorption profiles obtained from the SF formalism are certainly not perfect (at low density), we think that it is worthwhile to further investigate the method presented here, the more so because it is numerically simple.

Acknowledgment. We like to thank Frans Leermakers from the Department of Physical and Colloid Chemistry of the Wageningen Agricultural University in The Netherlands for the calculation of the adsorption profiles with the Scheutjens-Fleer self-consistent field theory. 